\title{
Extension of the clinical range of facioscapulohumeral dystrophy: report of six cases
}

\author{
A J van der Kooi, M C Visser, N Rosenberg, R van den Berg-Vos, J H J Wokke, E Bakker,
} $M$ de Visser

\begin{abstract}
Consensual diagnostic criteria for facioscapulohumeral dystrophy (FSHD) include onset of the disease in facial or shoulder girdle muscles, facial weakness in more than $50 \%$ of affected family members, autosomal dominant inheritance in familial cases, and evidence of myopathic disease in at least one affected member without biopsy features specific to alternative diagnoses.

Six patients did not meet most of these criteria but were diagnosed as FSHD by DNA testing, which showed small EcoRI fragments on chromosome 4q.

Their clinical signs and symptoms and results of auxiliary investigations are reported. The patients presented with foot extensor, thigh, or calf muscle weakness. None of them had apparent facial weakness, only one complained of weakness in the shoulders, none had a positive family history. Expert physical examination, however, showed a typical facial expression, an abnormal shoulder configuration on lifting the arms, or scapular winging. This raised the suspicion of FSHD, whereupon DNA analysis was done. In conclusion, the clinical expression of FSHD is much broader than indicated by the nomenclature. The possibility to perform DNA tests is likely to greatly expand the clinical range of FSHD.
\end{abstract}

(F Neurol Neurosurg Psychiatry 2000;69:114-116)

Keywords: facioscapulohumeral muscular dystrophy

Facioscapulohumeral muscular dystrophy (FSHD) is the third most common hereditary myopathy after Duchenne's and myotonic dys- trophy. It has a wide range of clinical manifestations typically starting in the second decade. In general, weakness initially involves the face and the periscapular muscles followed by the foot extensors, abdominal muscles, and the hip girdle. Other characteristics include striking asymmetric muscle involvement and sparing of the extraocular and bulbar muscles.

Workshops of the European Neuromuscular Centre have established the following diagnostic criteria to be used for genetic studies:(1) onset of the disease in facial or shoulder girdle muscles, sparing of the extraocular, pharyngeal, and lingual muscles and the myocardium; (2) facial weakness in more than $50 \%$ of the affected family members; (3) autosomal dominant inheritance in familial cases; (4) evidence of myopathic disease in EMG and muscle biopsy in at least one affected member without biopsy features specific to alternative diagnoses. ${ }^{2}$

In $1990^{3}$ linkage to a marker on chromosome $4 \mathrm{q}$ was found. Subsequently, affected people were shown to carry a small EcoRI fragment. ${ }^{4}$ The diagnosis of FSHD can be confirmed by DNA restriction fragment analysis in $95 \%$ of patients. ${ }^{5}$ EcoRI fragment size below $38 \mathrm{~kb}$ is compatible with a diagnosis of FSHD. ${ }^{6}$

In this report we describe six sporadic patients with an atypical presentation of FSHD giving rise to diagnostic difficulty. The diagnosis could only be established by DNA analysis.

\section{Patients}

The clinical features of the six patients are summarised in the table. Two patients are described in detail.

PATIENT 2

A 57 year old woman was referred to a neurologist in 1997 because she had had diffi-

20 January 2000

Accepted 6 March 2000

Table 1 Clinical features and data of ancillary investigations of six patients with FSHD

\begin{tabular}{|c|c|c|c|c|c|c|c|c|c|c|}
\hline Patient & Age/sex & Presenting symptoms & $\begin{array}{l}\text { Presenting } \\
\text { signs }\end{array}$ & $\begin{array}{l}\text { Age at onset } \\
\text { (y) }\end{array}$ & Progression & $C K$ & $C T$ & $E M G$ & $\begin{array}{l}\text { Muscle } \\
\text { biopsy }\end{array}$ & $\begin{array}{l}\text { EcoRI fragment } \\
\text { size }(k b)\end{array}$ \\
\hline 1 & $32 \mathrm{M}$ & $E^{\star}$ drop foot & $\mathrm{FS} \star \mathrm{E}^{\star}$ & 30 & $\mathrm{~m}$ & $2.5 \times$ & $\mathrm{T}^{\star} \mathrm{E}^{\star} \mathrm{C}^{\star}$ & $\mathrm{N}$ & NP & 30 \\
\hline 2 & $57 \mathrm{~F}$ & T: walking difficulty & FSHTC ${ }^{\star}$ & 50 & $\mathrm{~m}$ & $1.2 \times$ & $\mathrm{HTC}^{\star}$ & $\mathrm{N}$ & $\mathrm{N}$ & 26 \\
\hline 3 & $55 \mathrm{M}$ & C: inability to walk on toes & $\mathrm{FS}^{\star} \mathrm{T}^{\star} \mathrm{C}$ & 50 & $\mathrm{~m}$ & NI & $S^{\star} T^{\star} C^{\star}$ & $\mathrm{M}$ & NP & 20 \\
\hline 4 & $80 \mathrm{M}$ & $E^{\star}:$ drop foot & $\mathrm{S}^{\star} \mathrm{H}^{\star} \mathrm{E}^{\star}$ & 75 & $\mathrm{~m}$ & $2.5 \times$ & NP & NP & NP & 38 \\
\hline 5 & $65 \mathrm{M}$ & E: drop feet & $\mathrm{FS}^{\star} \mathrm{H}^{\star} \mathrm{E}^{\star}$ & 64 & $\mathrm{~m}$ & $3.5 \times$ & $\mathrm{S}^{\star} \mathrm{H}^{\star} \mathrm{CE} \mathrm{E}^{\star}$ & $\mathrm{M}$ & NP & 33 \\
\hline 6 & $40 \mathrm{~F}$ & $S^{\star}$ : shoulder pain & $\mathrm{S}^{\star} \mathrm{H}^{\star}$ & 36 & $\mathrm{~m}$ & NI & NP & M & NP & 38 \\
\hline
\end{tabular}

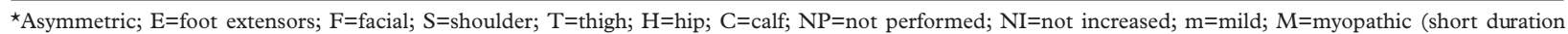
MUAPs); $\mathrm{N}=$ neurogenic (fibrillations, positive sharp waves, long duration MUAPs). 


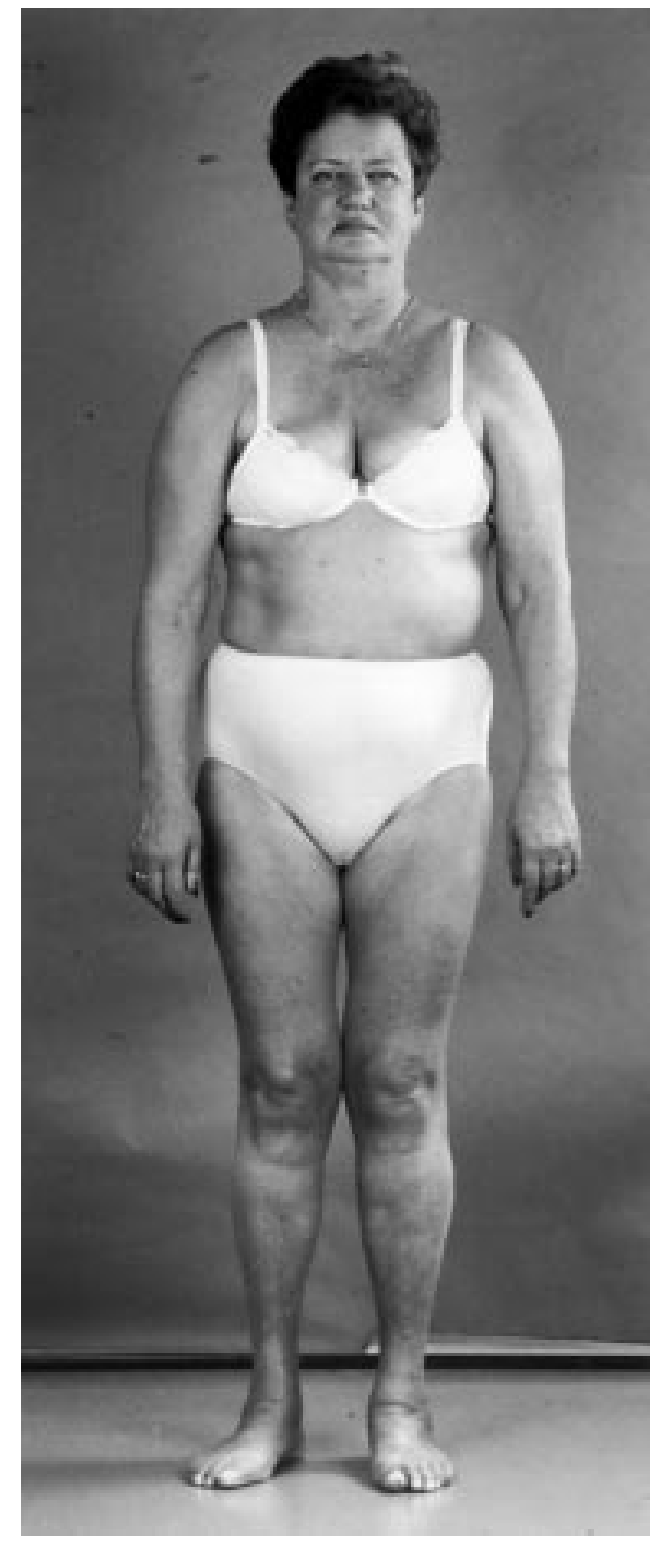

Patient 2 showing a myopathic face, and an abnormal shoulder configuration with atrophy of the lower part of the pectoralis major muscle (with permission).

culty walking for about 6 years. Neurological examination disclosed weakness of the hamstrings. An EMG showed fibrillations, positive sharp waves, and long duration polyphasic action potentials in the biceps femoris and gastrocnemius muscles which raised the suspicion of $\mathrm{S} 1$ radiculopathy. However, MRI of the lumbar spine was normal. Serum creatine kinase activity was slightly increased. Computed tomography of skeletal muscles demonstrated fatty degeneration in the posterior thigh and calf muscles. Muscle biopsy from the quadriceps muscle showed some atrophic fibres with increased non-specific esterase activity and a small endomysial mononuclear cellular infiltrate. Therefore, a tentative diagnosis of spinal muscular atrophy was made. The patient was then referred to the neuromuscular unit. On neurological examination a myopathic face, without clear weakness of the facial muscles (figure), an abnormal shoulder configura- tion, atrophy and weakness of the lower part of the pectoralis major muscle, some winging of the left scapula, and weakness of the gluteus maximus, hamstring, and right triceps surae muscles were found. A diagnosis of FSHD was suspected, and confirmed by DNA analysis. Family history was negative.

\section{PATIENT 3}

A 55 year old man was referred to a neurologist for foot pain and inability to walk on his toes for 4 to 5 years. On examination, thoracic kyphosis and mild weakness of the biceps and triceps brachii and calf muscles were demonstrated. Serum creatine kinase activity was normal. An EMG showed predominantly myopathic changes in the proximal arm and leg muscles.

The patient was referred to the neuromuscular centre. On examination he had no myopathic face but blowing his cheeks was not very forceful. Raising his arms disclosed an abnormal posture of the shoulders, and there was mild asymmetric weakness of both triceps brachii, and right deltoid muscles, and plantar flexors of the foot. Computed tomography of the skeletal muscles disclosed asymmetric fatty degeneration of latissimus dorsi, serratus anterior, rectus femoris, semimembranosus, and calf muscles. A diagnosis of FSHD was entertained, and confirmed by DNA analysis. Family history was negative.

\section{Discussion}

In this paper we describe six sporadic patients with genetically established FSHD, in whom presenting symptoms and signs initially caused substantial diagnostic confusion.

Three patients presented with foot extensor weakness, one with thigh weakness, one with inability to walk on his toes due to calf muscle involvement, and one with mild shoulder symptoms, such as tiredness and muscle pain. The clinical signs, however, were more extensive, and involved facial or shoulder involvement, whereas some degree of asymmetry was present in all cases. Facial involvement did not always imply overt facial weakness, but was sometimes only an abnormality of facial expression. All patients had an abnormal shoulder posture or a winging scapula on lifting their arms, unrecognised until specifically looked for.

According to the diagnostic criteria outlined by the European Neuromuscular Centre FSHD Consortium, onset of FSHD is either in the facial or shoulder girdle muscles. Facial weakness is the initial symptom in $20 \%$, but is reported to go unnoticed in up to $60 \% .^{8}$ Frequencies of scapulohumeral and pelvifemoral onset were $77 \%$ and $12 \%$, respectively. Padberg ${ }^{9}$ investigated a group of 107 patients, of whom 73 were symptomatic. The presenting symptoms were facial weakness in $10 \%$, shoulder weakness in $82 \%$, and foot extensor weakness in $8 \%$. None of his patients presented with pelvic girdle or calf muscle weakness. In the asymptomatic group of 34 patients, two showed only shoulder girdle weakness, one had shoulder weakness in combination with foot 
extensor involvement, and the other 31 had either facial weakness alone, or facial weakness in combination with shoulder girdle, foot extensor, or pelvic girdle weakness. Calf muscles are presumed to be affected only in later stages of the disease, ${ }^{10}$ although Patijn ${ }^{11}$ described early CT changes - that is, fatty degeneration of the medial head of the gastrocnemius muscle.

Onset is described as occurring between 3 and 44 years of age, mostly around the age of $15 .^{91012}$ In all our patients onset was much later, in four patients, beyond the age of 50 , in one at the age of 75 .

It is conceivable that FSHD is not recognised as such in many patients, in whom the initial symptoms and signs are not characteristically due to facioscapulohumeral muscular weakness. This is especially so if the family history is negative as in our patients. Our patients show that FSHD can present with walking difficulties due to drop foot, thigh, or calf muscle weakness. These atypical presentations gave rise to other diagnostic considerations. In cases of asymmetric foot extensor or foot flexor weakness radiculopathies or inclusion body myositis might be suspected. In cases of pelvifemoral weakness all possible causes for a limb girdle syndrome, including spinal muscular atrophy as in patient 2 might be considered, and in cases of scapula winging in the absence of facial weakness, any myopathy in which the shoulder girdle muscles are affected.

Ancillary investigations, such as assessment of creatine kinase activity, EMG, and muscle biopsy, often do not contribute to the diagnosis of FSHD, as in our patients. Creatine kinase activity is usually normal or only slightly increased, EMG may show sharp positive waves and fibrillations compatible with active denervation, and muscle biopsy shows either a non-specific myopathy, or atrophic, angular muscle fibres which are often considered to be of neurogenic origin. ${ }^{2}$ It takes careful examination, preferably by a neurologist who has specific expertise in neuromuscular diseases, to appreciate slight and often asymptomatic involvement, especially of facial and shoulder girdle muscles. Computed tomography of the skeletal muscles may be useful in demonstrating the asymmetric involvement of the muscles. Subsequent DNA analysis can then be undertaken to confirm or exclude the diagnosis of FSHD based on clinical considerations. In all our patients small EcoRI fragments were detected, in two of them shorter than $30 \mathrm{~kb}$. Although an inverse correlation between frag- ment size and age at onset has been shown ${ }^{13}$ this does not apply to our patients in whom onset was at the age of 50 years. Furthermore, an association between fragment size and disease severiy has been described. ${ }^{14}{ }^{15}$ Sporadic cases are reported to have significantly smaller fragment sizes (13-24 kb) than familial cases, and as a group they were more severely affected compared with familial cases of FSHD. ${ }^{16}$ Lunt et al argued that this finding could be due to ascertainment bias as a de novo mutation is only recognised when this has caused a severe phenotype. All our patients were sporadic, and had a mild phenotype, in the presence of fragments from 20-38 kb. Whether all our patients are really de novo mutations remains unclear as none of the parents had been available for DNA analysis.

1 Tawil R, Figlewicz DA, Griggs RC, et al. Facioscapulohumeral dystrophy: a distinct regional myopathy with a novel molecular pathogenesis. Ann Neurol 1998;43:279-82.

2 Padberg GW, Lunt PW, Koch M, et al. Facioscapulohumeral muscular dystrophy. In: Emery AEH, ed. Diagnostic criteria muscular dystrophy. In: Emery AEH, ed. Diagnostic criteria of Medicine, 1997:9-15.

3 Wijmenga C, Frants RR, Brouwer OF, et al. Localisation of facioscapulohumeral muscular dystrophy gene on chromofacioscapulohumeral muscular dy
some 4. Lancet 1990;336:651-3.

4 Wijmenga C, Hewitt JE, Sandkuijl LA, et al. Chromosome Wq DNA rearrangements associated with facioscapulohumeral muscular dystrophy. Nat Genet 1992;2:26-30.

5 Upadhyaya M, Maynard J, Rogers MT, et al. Improved molecular diagnosis of facioscapulohumeral muscular dystrophy (FSHD): validation of the differential double digestion for FSHD. f Med Genet 1997;34:476-9.

6 Orrell RW, Tawil R, Forrester J, et al. Definitive molecular diagnosis of facioscapulohumeral dystrophy. Neurology 1999;52:1822-6.

7 Lemmerts RJ, Van der Maarel SM, Van Deutekom JC, et al. Inter- and intrachromosomal subtelomeric rearrangements on 4q35: implications for facioscapulohumeral muscular dystrophy (FSHD) aetiology and diagnosis. Hum Molec Genet 1998;7:1207-14.

8 Chung CS, Morton NE. Discrimination of genetic entities in muscular dystrophy. Am f Hum Genet 1959;11:339-59. in muscular dystrophy. Am f Hum Genet 1959;11:339-59.
Padberg G. Facioscapulohumeral disease [thesis]. University Padberg G. Facioscapulohumeral disease
of Leiden, The Netherlands, 1982.

10 Tyler FH, Stephens FE. Studies in disorders of muscle. II. Clinical manifestations and inheritance of facioscapulohumeral dystrophy in a large family. Ann Intern Med 1999; 32:640-60.

11 Patijn J. De ziekte van Landouzy-Dejerine [thesis]. University of Amsterdam, The Netherlands, 1983.

12 Walton JN, Nattrass FJ. On the classification, natural history and treatment of the myopathies. Brain 1954;77:169-231.

13 Lunt PV, Jardine PE, Koch MC, et al. Correlation between fragment size at D4F104S1 and age at onset or at wheelchair use, with a possible generational effect, accounts for much phenotypic variation in effect, accounts for much phenotypic variation in 4q35Molecul Genet 1995;4:951-8.

14 Ricci E, Galluzzi G, Deidda G, et al. Progress in the molecular diagnosis of facioscapulohumeral muscular dystrophy and correlation between the number of KpnI repeats at the and correlation between the number of KpnI repeats at the 4q35 locus.

15 Tawil R, Forrester J, Griggs RC, et al. Evidence for anticipation and association of deletion size with severity in facioscapulohumeral muscular dystrophy. Ann Neurol 1996;39:744-8.

16 Lunt PW, Jardine PE, Koch M, et al. Phenotypic-genotypic correlation will assist genetic counseling in 4q35facioscapulohumeral muscular dystrophy. Muscle Nerve 1995;(suppl 2):S103-9. 